\title{
Pensamento Computacional: Panorama dos Grupos de Pesquisa no Brasil
}

\author{
Rozelma Soares de França ${ }^{1,2}$, Patrícia Cabral de Azevedo Restelli Tedesco ${ }^{1}$ \\ ${ }^{1}$ Centro de Informática - Universidade Federal de Pernambuco (UFPE) \\ 50740-560 - Recife - PE - Brasil \\ ${ }^{2}$ Departamento de Educação - Universidade Federal Rural de Pernambuco (UFRPE) \\ 52171-900 - Recife - PE - Brasil \\ \{rsf2, pcart\}@cin.ufpe.br
}

\begin{abstract}
Computational thinking has been considered one of the key competencies to thrive in a technological world, and a number of initiatives have emerged aimed at its development from basic education. In this context, this research analyzes the Directory of Research Groups of CNPq, focusing on research lines of computational thinking, aiming to characterize research on the subject in Brazil. Among other findings, it was identified that the groups are from diverse areas, but the greatest concentration is in Computing. The results reported may support the intercommunication between educators, students and researchers interested in the subject, as well as the planning of strategic educational actions for the country.
\end{abstract}

Resumo. O pensamento computacional tem sido considerado uma das principais competências para prosperar em um mundo tecnológico, e diversas iniciativas têm surgido visando o seu desenvolvimento desde a educação básica. Neste contexto, esta pesquisa analisa o Diretório de Grupos de Pesquisa do CNPq, focando em linhas de pesquisa de pensamento computacional, visando caracterizar a pesquisa sobre o tema no Brasil. Entre outros achados, identificouse que os grupos são de áreas diversificadas, mas a maior concentração está na Computação. Os resultados reportados poderão subsidiar a intercomunicação entre educadores, estudantes e pesquisadores interessados no tema, como também o planejamento de ações educacionais estratégicas para o país.

\section{Introdução}

O termo Pensamento Computacional (PC) se popularizou com o artigo de Wing (2006), embora tenha sido cunhado por Papert (1980) em seu livro Mindstorms: Children, Computers, And Powerful Idea. Desde então diversas definições para o termo têm sido propostas, estando a ênfase na resolução de problemas, explorando conceitos fundamentais de Ciência da Computação (CC). À essa narrativa, Barba (2016) faz críticas por acreditar que há um desvio da ideia original e poderosa de Seymour Papert, há mais de 30 anos. Para a autora, o aspecto operacional de tornar os problemas computáveis é essencial, mas não aspiracional. Ela enfatiza ainda que a maioria das pessoas não quer ser um cientista da computação, mas todos podem usar computadores como uma extensão de sua mente, para experimentar e criar coisas que são importantes para as pessoas. 
VIII Congresso Brasileiro de Informática na Educação (CBIE 2019)

Anais do XXX Simpósio Brasileiro de Informática na Educação (SBIE 2019)

Para a Computer Science Teachers Association (CSTA, 2011), em um mundo cada vez mais tecnológico, é necessário que os estudantes tenham um claro entendimento dos princípios e práticas da $\mathrm{CC}$, independente de seus campos de estudo e atuação. $\mathrm{O}$ acesso ao conhecimento dos fundamentos de CC pode auxiliar a utilizar tecnologias de maneira mais consciente, além de apoiar a criação de sistemas de computação para melhorar a qualidade de vida das pessoas (ibid).

Nesse contexto, para além da codificação, a essência do PC deve ser o que se pode fazer enquanto interage-se com computadores, como extensões da mente humana, para criar e descobrir (BARBA, 2016). Sob essa ótica, a habilidade de PC pode favorecer profissionais de diferentes áreas que poderão utilizar a Computação como representação do conhecimento para os ajudar a pensar.

A temática tem despertado o interesse das comunidades científica e escolar, como também de chefes de Estado, que têm buscado promover o PC por meio de diferentes iniciativas. Uma delas é a Computer Science For All ${ }^{1}$ anunciada por Obama, à época presidente dos Estados Unidos, visando formar estudantes americanos em habilidades computacionais de que precisam para prosperar em uma economia digital.

No Brasil é possível observar iniciativas realizadas em diferentes regiões do país e publicadas em anais de eventos do Congresso Brasileiro de Informática na Educação (CBIE), como o Workshop de Ensino em Pensamento Computacional, Algoritmos e Programação (WAlgProg). Há ainda aquelas publicadas nos anais do Workshop sobre Educação em Computação (WEI) e em periódicos como a Revista Brasileira de Informática na Educação (RBIE) e a Revista Novas Tecnologias na Educação (RENOTE).

$\mathrm{Na}$ forma de políticas públicas, apesar de tímida, houve uma conquista publicada na Base Nacional Comum Curricular (MEC, 2018) que inclui PC no trato de conteúdos da área de Matemática. Há ainda as "Diretrizes para ensino de Computação na Educação Básica" propostas pela Sociedade Brasileira de Computação (SBC, 2019). O documento define competências e habilidades da Educação Infantil até o Ensino Médio contemplando três eixos: Pensamento Computacional, Mundo Digital e Cultura Digital.

Também, o Centro de Inovação para a Educação Brasileira (CIEB, 2018) elaborou uma proposta que atende da Educação Infantil ao Ensino Fundamental II. O documento, intitulado "Currículo de Referência em Tecnologia e Computação", tem como objetivo oferecer diretrizes e orientações de apoio às redes de ensino e escolas para incluir os temas tecnologia e computação em suas propostas curriculares.

Diante desse cenário, buscou-se traçar um panorama das pesquisas em PC no Brasil. O estudo aqui reportado é um levantamento abrangente de todos os grupos e linhas de pesquisa que investigam PC, em diferentes áreas do conhecimento. A análise teve como base o Diretório dos Grupos de Pesquisa no Brasil (DGP) da Plataforma Lattes do Conselho Nacional de Desenvolvimento Científico e Tecnológico $(\mathrm{CNPq})$ e os resultados obtidos são apresentados e discutidos ao longo deste artigo.

\footnotetext{
${ }^{1}$ Disponível em <https://obamawhitehouse.archives.gov/blog/2016/01/30/computer-science-all>.
} 
VIII Congresso Brasileiro de Informática na Educação (CBIE 2019)

Anais do XXX Simpósio Brasileiro de Informática na Educação (SBIE 2019)

\section{Estudos secundários sobre pensamento computacional}

Na literatura, há diversos estudos secundários que tratam de PC. Analisando o portal de publicações da Comissão Especial de Informática na Educação (CEIE) ${ }^{2}$ é possível localizar diferentes interesses como foco dessas investigações.

De forma mais abrangente, Ortiz e Pereira (2018) buscaram identificar iniciativas para promover PC mapeando bases nacionais e internacionais. Bordini et al. (2017) focaram a pesquisa nos ensinos fundamental e médio. E Fernandes e Silveira (2016) estavam interessados em iniciativas para o desenvolvimento de PC por meio da modalidade de ensino a distância.

O desenvolvimento de PC por meio do ensino de programação foi interesse de Zanetti et al. (2016) e Rodrigues et al. (2018), sendo que estes últimos focaram na computação desplugada como abordagem de ensino. O mapeamento de ações que usaram robótica (AVILA et al., 2017), jogos digitais (ZABOT et al., 2018), objetos de aprendizagem (CARVALHO et $a l ., 2017)$ também tem sido contemplado. Nesse contexto, um dos trabalhos localizados foi o de Bombasar et al. (2015) que visaram identificar as principais ferramentas utilizadas no ensino de PC entre 2006 e 2015. Dentre os resultados, não foram identificadas ferramentas baseadas nas teorias e formalismos desenvolvidos por Alan Turing, o pai da computação moderna.

A avaliação de PC também tem sido foco dos estudos secundários nacionais, como pode ser visto em Avila et al. (2017) e Araújo et al. (2016). Há interesse também no mapeamento de ações que considerem o contexto sociocultural dos estudantes (ORTIZ et al., 2018). A interdisciplinaridade do PC (NASCIMENTO et al., 2018) e a relação dessa habilidade computacional com a Matemática (BARCELOS et al., 2015) também tem norteado algumas pesquisas.

Diante do cenário apresentado, este estudo é inédito por traçar o perfil dos grupos de pesquisa sobre PC no Brasil, destacando, dentre outros, o perfil dos pesquisadores, a área e a finalidade das linhas de pesquisa no país. Estes dados são importantes uma vez que a discussão sobre PC é interdisciplinar e complexa, fazendo-se necessárias teorias e práticas sob a ótica de diferentes áreas.

\section{Procedimentos metodológicos}

Nesta pesquisa utilizou-se a base de dados disponibilizada pelo Diretório dos Grupos de Pesquisa no Brasil, integrante da Plataforma Lattes do Conselho Nacional de Desenvolvimento Científico e Tecnológico.

O levantamento dos grupos de pesquisa foi feito utilizando-se a palavra-chave pensamento computacional no campo termo de busca do site de busca parametrizada da Base Corrente do DGP, com os filtros busca exata e consulta por grupo e com aplicação do termo nos campos nome do grupo, linha de pesquisa e palavra-chave da linha de pesquisa, em 21 de maio de 2019. Foram considerados os grupos certificados e não-atualizados, resultando em um total de 28 grupos. O mesmo procedimento foi usado para a busca da palavra-chave raciocínio computacional, porém nenhum dado foi retornado. O passo seguinte consistiu na definição dos perfis desses grupos, em termos de distribuição geográfica, maturidade, formação acadêmica dos pesquisadores e área do conhecimento predominante.

Com o objetivo de fazer o recorte das linhas de pesquisa de pensamento computacional, buscou-se tal termo nos títulos e palavras-chave das linhas de pesquisa dos 28 grupos retornados. O passo seguinte consistiu na identificação da quantidade de pesquisadores e

\footnotetext{
${ }^{2}$ Disponível em $<$ http://www.br-ie.org/pub/index.php/index $>$.
} 
VIII Congresso Brasileiro de Informática na Educação (CBIE 2019)

Anais do XXX Simpósio Brasileiro de Informática na Educação (SBIE 2019)

estudantes envolvidos na linha sobre o tema, além da finalidade das pesquisas. Para classificação das finalidades das linhas de pesquisa, levou-se em consideração as seguintes categorias: Teoria, Metodologia, Desenvolvimento, Avaliação e Aplicação (QUEIROZ et al., 2017). Os dados levantados foram analisados e são reportados na seção seguinte.

\section{Resultados e discussões}

A lista dos 28 grupos de pesquisa sobre PC analisados pode ser consultada no link $<$ http://bit.ly/GruposPesquisaPC $>$. Nesta seção, serão apresentadas representações gráficas dos dados, como também discussões a partir do panorama traçado que poderão nortear mais investigações sobre o tema no Brasil.

\subsection{Distribuição geográfica}

A região brasileira com maior número de grupos de pesquisa sobre PC é a Sudeste, com 13 grupos. Já as regiões Centro-Oeste e Norte são as com menor número, com um e dois grupos respectivamente. Embora todas as regiões brasileiras apresentem grupos de pesquisa sobre PC, as regiões Sudeste e Sul juntas possuem maior representativa, com 67,85\% (19 grupos) do total. Ainda é possível notar que o Nordeste e o Sul concentram o mesmo número de grupos, seis, conforme Figura 1.

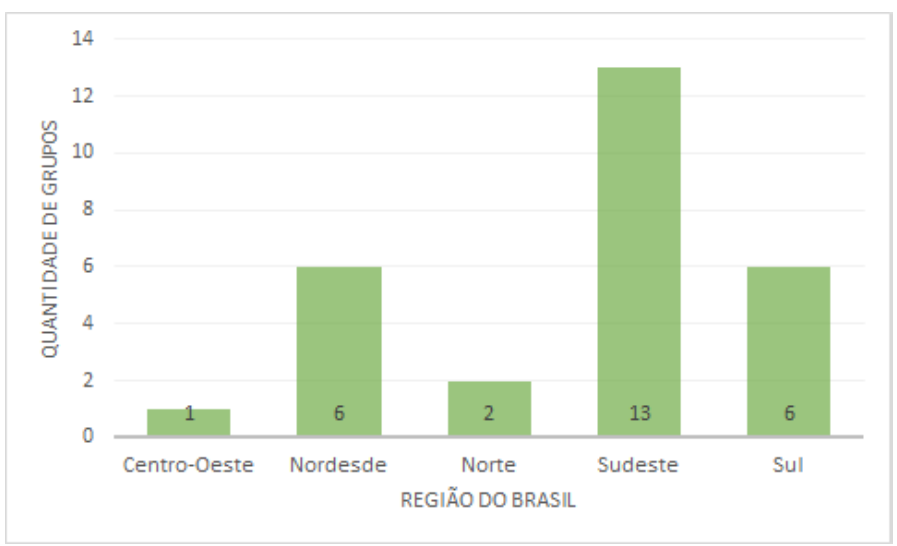

Figura 1. Distribuição geográfica dos grupos de pesquisa

Tais resultados, em boa parte, estão em consonância com os encontrados por Silva et al. (2018) ao buscarem identificar estratégias para os processos de ensino e aprendizagem do pensamento computacional no Brasil. No estudo, os autores utilizaram o buscador do portal de publicações da CEIE, como também da RENOTE ${ }^{3}$.

Um dos achados do estudo de Silva et al. (2018) foi quanto ao número de estudos por região brasileira: a maior concentração foi no Sul; diferente desta pesquisa que observou maior concentração de grupos de estudos na região Sudeste. Por outro lado, Sudeste e Sul juntos também têm a maior representativa. No trabalho supracitado, identificou-se que o Nordeste ocupa o segundo lugar quanto à pesquisa sobre PC. Esses resultados estão em conformidade com esta investigação, sendo o segundo lugar ocupado pelo Nordeste e pelo Sul quanto ao número de grupos de pesquisa por região. Centro-Oeste e Norte aparecem na retaguarda em ambos os estudos.

\footnotetext{
${ }^{3}$ Disponível em $<$ https://seer.ufrgs.br/renote/ $>$.
} 
VIII Congresso Brasileiro de Informática na Educação (CBIE 2019)

Anais do XXX Simpósio Brasileiro de Informática na Educação (SBIE 2019)

\subsection{Maturidade}

O grupo mais antigo com linha de pesquisa em PC foi formado em 1993, mas só a partir de 2010 o interesse pelo tema vem crescendo, tendo em 2017 um aumento significativo, como mostra a Figura 2.

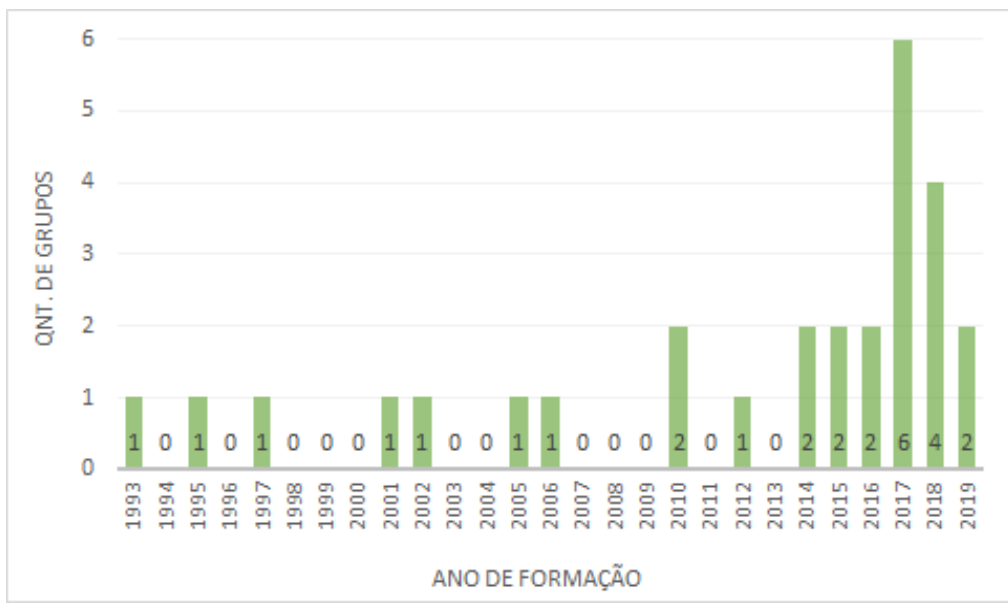

Figura 2. Ano de formação dos grupos de pesquisa

É importante destacar que o DGP não fornece dados sobre a data de criação das linhas de pesquisa, apenas do grupo; não sendo possível concluir a partir desses dados que em 1993 o grupo identificado já realizava pesquisa sobre PC no Brasil.

O termo pensamento computacional se popularizou com um artigo de Jeannette Wing em 2006. No entanto, como mencionado anteriormente, já em 1980 Papert usou tal termo. A narrativa construída por Wing (2006) defende o PC como uma habilidade fundamental para todos e não apenas para os cientistas da computação, sendo um dos pilares fundamentais da formação básica, junto com a leitura, a escrita e a aritmética.

Provavelmente o artigo teórico de Wing (2006) tenha sido um fator que incentivou e potencializou esforços de pesquisa na área de $\mathrm{PC}$, como a criação de grupos de pesquisa no Brasil e no exterior. Além disso, no Brasil, em 2015, foi criado o WAlgProg ${ }^{4}$ em resposta à ausência de um evento nacional que concentrasse pesquisas e discussões sobre o ensino de Pensamento Computacional, Algoritmos e Programação. Face à sua aceitação pela comunidade científica, o evento tem sido realizado anualmente como um dos workshops do CBIE possibilitando intercomunicação entre pesquisadores, docentes, discentes e demais interessados. Este dado pode justificar o aumento da criação de grupos com linhas de pesquisa sobre PC nos últimos anos no país.

\subsection{Formação acadêmica}

Os grupos de pesquisa sobre PC têm em sua maioria pesquisadores doutores $(59,5 \%)$, como pode ser visto na Figura 3. Essa tendência não é percebida no grupo de estudantes que apresenta maior número no nível de graduação $(39,5 \%)$. Também é possível identificar a participação de técnicos e colaboradores estrangeiros nos grupos de pesquisa analisados. No caso dos técnicos, sua formação varia entre mestrado, especialização e graduação. Já os colaboradores estrangeiros têm doutorado em sua totalidade.

\footnotetext{
${ }^{4}$ Disponível em $<$ http://walgprog.gp.utfpr.edu.br/>
} 
VIII Congresso Brasileiro de Informática na Educação (CBIE 2019)

Anais do XXX Simpósio Brasileiro de Informática na Educação (SBIE 2019)

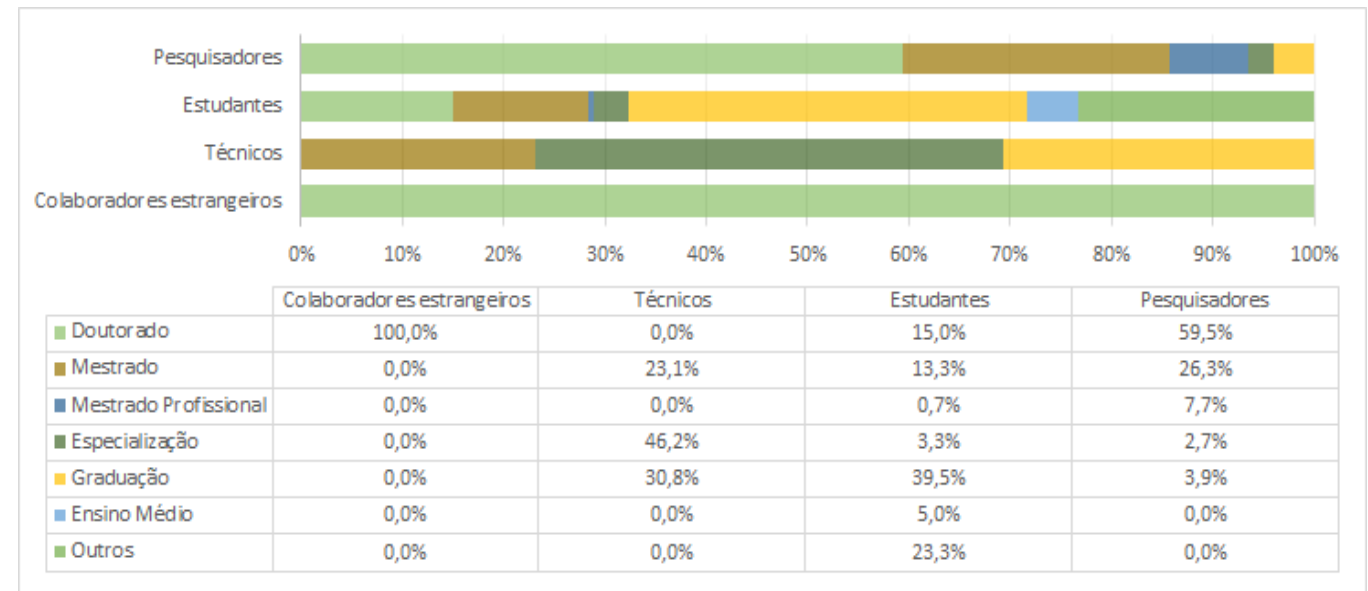

Figura 3. Formação dos participantes dos grupos de pesquisa

Observando a Figura 4, é possível perceber que do total de participantes dos grupos de pesquisa sobre PC, a maior parte deles é da categoria estudantes; aproximadamente 52\%, seguido dos pesquisadores, técnicos e colaboradores estrangeiros, nesta ordem. Este resultado pode apontar para a necessidade de pesquisas nacionais com colaboração de pesquisadores de outros países, como estratégia para aprender, conceber e implementar políticas de ensino de Ciência da Computação na educação básica, como as existentes em países como Austrália, Estados Unidos, Israel e Reino Unido.

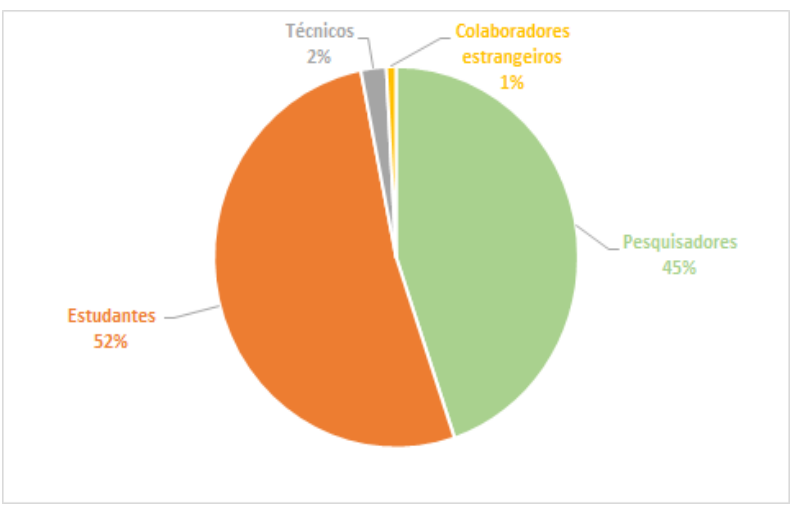

\section{Figura 4. Percentual de participantes dos grupos de pesquisa por categoria}

Os grupos analisados possuem linhas de pesquisa diversas, dentre elas aquelas relacionadas a PC. Assim, foi feito um recorte das linhas de pesquisa que tratam especificamente sobre PC e comparada a quantidade de estudantes e pesquisadores dessas linhas com a quantidade total de participantes do grupo de pesquisa, nessas categorias. Os resultados dessa tarefa são apresentados nas Figuras 5a e 5b.

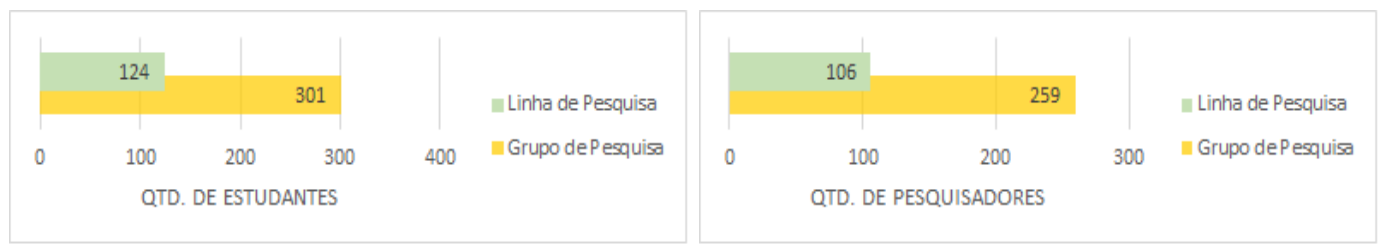

Figure 5. Quantidade de: a) estudantes e b) pesquisadores nos grupos e linhas de pesquisa de PC 
Como pode ser observado, aproximadamente $41 \%$ dos estudantes dos grupos atuam em linhas de pesquisas de PC. Esse número é um pouco menor no caso dos pesquisadores onde é possível constatar a presença de aproximadamente $40 \%$ deles do total de pesquisadores do grupo.

\section{4. Área}

Quanto ao domínio de conhecimento dos grupos de pesquisa sobre PC, identificou-se que a maior parte deles é da área de Computação (57\%), seguida da área de Educação com 29\%, conforme pode ser observado na Figura 6. Outras áreas também aparecem na classificação, porém em menor proporção. São elas: Arquitetura e Urbanismo, Administração e Física.

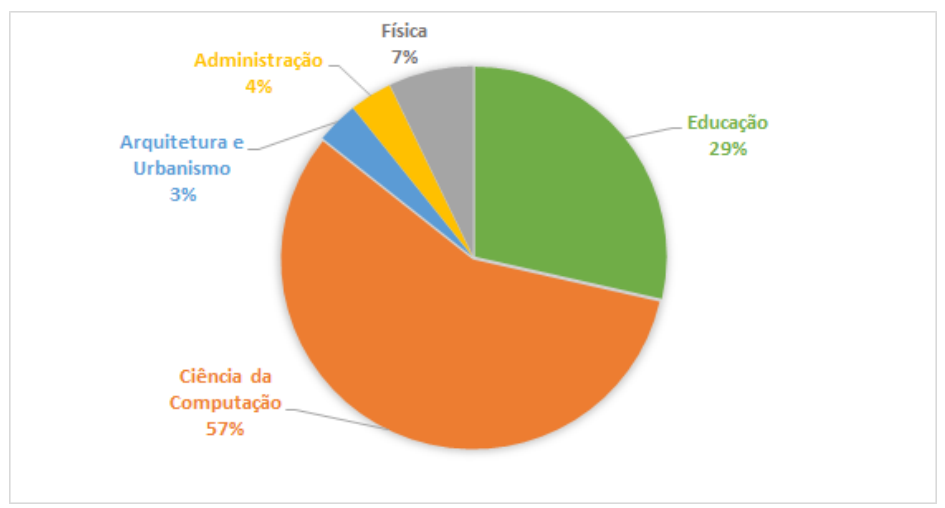

Figura 6. Área dos grupos de pesquisa

Pela dimensão que o incentivo à prática de programação por não-programadores tomou; tal como a Hora do Código ${ }^{5}$, atrelado à busca de talentos para a Computação, não é surpreende que a área de Computação tenha tido maior percentual de grupos de pesquisa sobre PC. É esperado que pesquisadores e profissionais da área se engajem em ações que repercutam em seus nichos.

Como visto na Figura 6, as propostas de soluções aos desafios da área, em geral, são desenvolvidas por pesquisadores da área da Computação. No entanto, é importante ressaltar que há questões pedagógicas e de Psicologia que devem ser consideradas, o que torna o PC um campo interdisciplinar e complexo. Assim, desde a sua concepção até métodos que deem suporte ao seu desenvolvimento e avaliação é acertado incluir pesquisadores de outras áreas nessas atividades.

Segundo Wing (2006), PC é a base da Ciência da Computação podendo ser aplicado na resolução de problema de outras áreas, como Jornalismo (WOLZ et al., 2010) e Biologia (PRIAMI, 2007). Nesse contexto, é interessante notar o engajamento de grupos de pesquisa nacionais, que não são da área de Computação, em linhas de pesquisa que tratem da aplicação de PC em suas áreas. Este fator pode possibilitar que tais grupos se beneficiem de métodos e técnicas empregadas na Computação na resolução de seus problemas profissionais e cotidianos.

\subsection{Finalidades das linhas de pesquisa}

Quanto à finalidade das linhas de pesquisa de PC, a maioria é destinada à Metodologia (32\%), como pode ser visto na Figura 7. Ainda é possível perceber o interesse por aspectos teóricos (22\%), pelo desenvolvimento de tecnologias que deem suporte ao ensino (14\%), como também

\footnotetext{
${ }^{5}$ Disponível em <https://hourofcode.com/br> Acesso em 31 de maio de 2019.
} 
VIII Congresso Brasileiro de Informática na Educação (CBIE 2019)

Anais do XXX Simpósio Brasileiro de Informática na Educação (SBIE 2019)

a aplicação de PC (18\%). Não foram identificadas linhas de pesquisa que tratem explicitamente sobre a avaliação de aspectos de PC na descrição de suas finalidades.

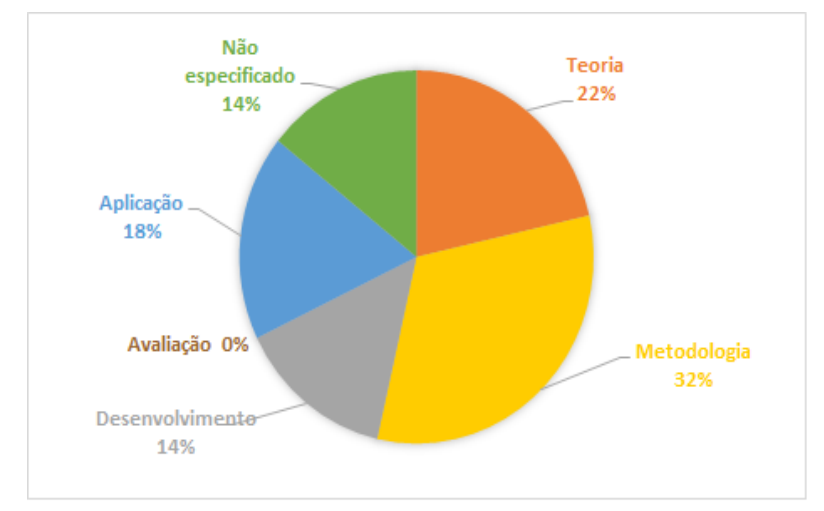

Figura 7. Finalidade das linhas de pesquisa

Em relação à teoria, um dos caminhos que podem ser seguidos pelos pesquisadores diz respeito à compreensão do que é $\mathrm{PC}$ e a necessidade de seu desenvolvimento desde a educação básica. Nesse sentido, Wing (2006) enfatiza que PC não se restringe ao simples uso de técnicas de programação. A partir de sua definição outras surgiram para o termo. Uma delas foi proposta pela International Society for Technology in Education (ISTE) em colaboração com a Computer Science Teachers Association (CSTA) e a National Science Foundation (NSF), a qual embute os seguintes conceitos em sua definição: coleta de dados, análise de dados, representação de dados, decomposição de problemas, abstração, algoritmos e procedimentos, automação, simulação e paralelismo.

Outras enfatizam que antes que os computadores sejam usados para resolver um problema, o problema em si e as formas como ele poderia ser resolvido devem ser entendidos, e quatro técnicas de PC conhecidas como decomposição, reconhecimento de padrões, abstração e algoritmos podem ajudar nessas tarefas (BBC, 2015). Apesar de considerar valiosas, há críticas às definições de PC como uma habilidade com ênfase na resolução de problemas, que podem desviar da ideia proposta por Papert (BARBA, 2016).

Em todos os casos, considera-se que os grupos de pesquisa nacionais podem explorar esse campo de investigação, buscando melhor compreender o PC e seus desdobramentos. Tais achados poderão nortear a definição do que, quando e como ensinar e avaliar PC nos diversos níveis de ensino.

\section{Considerações Finais}

Esta pesquisa mapeou os grupos de pesquisa sobre PC no Brasil a partir dos dados disponibilizados no DGP/CNPq. Com os resultados obtidos, conclui-se que nos últimos anos houve um aumento do interesse sobre PC no país, demonstrado pela criação de grupos de pesquisa com linhas voltadas à temática. Apesar dos grupos serem de áreas diversificadas, a maior parte é da Computação. Além disso, há uma concentração de grupos de pesquisa no Sudeste, em detrimento do Centro-Oeste e Norte. Estes resultados sugerem a necessidade de expansão dos grupos para outras áreas do conhecimento, como também para outras regiões do país.

Apesar da expansão da pesquisa sobre PC nos últimos anos, ainda são muitos os desafios para sua implementação na educação básica, como também no ensino superior. Esses desafios 
VIII Congresso Brasileiro de Informática na Educação (CBIE 2019)

Anais do XXX Simpósio Brasileiro de Informática na Educação (SBIE 2019)

perpassam a concepção de PC, como também métodos que deem suporte ao seu desenvolvimento e avaliação. Analisando os grupos de PC nacionais, identificou-se que metodologias de ensino têm sido uma das finalidades de pesquisa. A avaliação dessa habilidade computacional, no entanto, não foi contemplada, podendo ser foco de futuras investigações.

\section{Referências}

Araújo, A. L. S. O., Andrade, W. L., Guerrero, D. D. D. (2016) “Um Mapeamento Sistemático sobre a Avaliação do Pensamento Computacional no Brasil". In: Anais dos Workshops do V Congresso Brasileiro de Informática na Educação.

Avila, C., Cavalheiro, S., Bordini, A., Marques, M., Cardoso, M., Feijó, G. (2017) "Metodologias de avaliação do Pensamento Computacional: uma revisão sistemática". In: Anais do XXVIII Simpósio Brasileiro de Informática na Educação.

Avila, C., Cavalheiro, S., Bordini,, A., Marques, M. (2017) "O Pensamento Computacional por meio da Robótica no Ensino Básico - Uma Revisão Sistemática”. In: Anais do XXVIII Simpósio Brasileiro de Informática na Educação.

Barba, L. A. (2016), "Computational Thinking: I do not think it means what you think it means". Disponível em <https://lorenabarba.com/blog/computational-thinking-i-do-not-think-itmeans-what-you-think-it-means/>. Acesso de 31 de maio de 2019.

Barcelos, T., Muñoz, R., Villarroe, R., Silveira, I. (2015) "Relações entre o Pensamento Computacional e a Matemática: uma Revisão Sistemática da Literatura". In: Anais dos Workshops do IV Congresso Brasileiro de Informática na Educação.

BBC (2015) "What is computational thinking?". Disponível em $<$ http://www.bbc.co.uk/education/guides/zp92mp3/revision>. Acesso em 31 de maio de 2019.

Bombasar, J. R., Santiago, R., Miranda, E. M., Raabe, A. L. A. (2015) "Ferramentas para o Ensino-Aprendizagem do Pensamento Computacional: onde está Alan Turing?". In: Anais do XXVI Simpósio Brasileiro de Informática na Educação.

Bordini, A., Avila, C., Cunha, M., Cavalheiro, S., Foss, L. (2017) "Pensamento Computacional nos ensinos fundamental e médio: uma revisão sistemática". In: Anais do XXVIII Simpósio Brasileiro de Informática na Educação".

Carvalho, J. M., Netto, J. F. M., Almeida, T. O. (2017) "Revisão Sistemática de Literatura sobre o Pensamento Computacional por Meio de Objetos de Aprendizagem”. In: Anais do XXVIII Simpósio Brasileiro de Informática na Educação.

CIEB - Centro de Inovação para a Educação Brasileira. (2018) "Currículo de Referência em Tecnologia e Computação - Da Educação Infantil ao Ensino Fundamental”. Disponível em $<$ http://curriculo.cieb.net.br/>. Acesso em 31 de maio de 2019.

CSTA - Computer Science Teacher Association. (2011) "CSTA K-12 Computer Science Standards. CSTA Standards Task Force”. ACM - Association for Computing Machinery.

Fernandes, H. B., Silveira, I. F. (2016) "Pensamento Computacional: iniciativas para o seu desenvolvimento por meio da modalidade de Ensino a Distância". In: Anais dos Workshops do V Congresso Brasileiro de Informática na Educação.

ISTE - International Society for Technology in Education; CSTA - Computer Science Teachers Association; NSF - National Science Foundation. (2011) "Computational thinking: leadership toolkit". First Edition. 
VIII Congresso Brasileiro de Informática na Educação (CBIE 2019)

Anais do XXX Simpósio Brasileiro de Informática na Educação (SBIE 2019)

MEC - Ministério da Educação (2018), "Base Nacional Comum Curricular - Educação é a Base". Disponível

em $<$ http://basenacionalcomum.mec.gov.br/images/BNCC_EI_EF_110518_versaofinal_site.p df $>$ Acesso em 31 de maio de 2019.

Nascimento, C. A., Santos, D. A., Tanzi, A. (2018) "Pensamento Computacional e Interdisciplinaridade na Educação Básica: um Mapeamento Sistemático". In: Anais dos Workshops do VII Congresso Brasileiro de Informática na Educação.

Ortiz, J. S. B, Moreira, C., Pereira, R. (2018) “Aspectos do Contexto Sociocultural dos Alunos estão Presentes nas Pesquisas para Ensinar Pensamento Computacional?". In: Anais dos Workshops do VII Congresso Brasileiro de Informática na Educação.

Ortiz, J. S. B., Pereira, R. (2018) "Um Mapeamento Sistemático sobre as Iniciativas para Promover o Pensamento Computacional". In: Anais do XXIX Simpósio Brasileiro de Informática na Educação.

Papert, S. (1980). "Mindstorms: Children, Computers, And Powerful Ideas", Basic Books.

Priami, C. (2007) "Computational Thinking in Biology". In: Priami C. (eds) Transactions on Computational Systems Biology VIII. Lecture Notes in Computer Science, vol 4780. Springer, Berlin, Heidelberg.

Queiroz, A. C. M., Tori, R., Nascimento, A. M. (2017) "Realidade Virtual na Educação: Panorama dos Grupos de Pesquisa no Brasil”. In: Anais do XXVIII Simpósio Brasileiro de Informática na Educação.

Rodrigues, S. R. C., Aranha, E., Silva, T. R. (2018) “Computação Desplugada no Ensino de Programação: Uma Revisão Sistemática da Literatura”. In: Anais do XXIX Simpósio Brasileiro de Informática na Educação.

SBC - Sociedade Brasileira de Computação (2019), "Diretrizes para ensino de Computação na Educação Básica”. Disponível em < http://www.sbc.org.br/educacao/diretrizes-para-ensino-de-computacao-na-educacaobasica $>$. Acesso em 31 de maio de 2019.

Silva, K. S., Pereira, N. P., Odakura, V. (2018) "Mapeamento Sistemático: estratégias para o ensino-aprendizagem do Pensamento Computacional no Brasil”. In: XXIII Conferência Internacional sobre Informática na Educação.

Wing, J. M. (2006). “Computational thinking”, Communications of the ACM, v. 49, n. 3, p. 33.

Wolz, Ursula et al. (2010) "Computational thinking via interactive journalism in middle school". In: Proceedings of the 41st ACM Technical Symposium on Computer Science Education.

Zabot, D., Andrade, S. R., Matos, E. S. (2018) "Raciocínio Computacional e Jogos Digitais: categorias e mecânicas”. In: Anais do XXIX Simpósio Brasileiro de Informática na Educação.

Zanetti, H. A. P., Borges, M. A. F., Ricarte, I. L. M. (2016) "Pensamento Computacional no Ensino de Programação: Uma Revisão Sistemática da Literatura Brasileira”. In: Anais do XXVII Simpósio Brasileiro de Informática na Educação. 